\title{
RAPID ESTIMATIONS OF BIOLOGICAL FINENESS OF COTTON FIBERS USING MICROMAT DATA
}

\author{
Mohamed $^{1}$, A.A.; M.G. Sief ${ }^{1}$ and S.H. El-Hariry' \\ 1. Cotton Research Institute, Agriculture Research Center, Giza, Egypt
}

Keywords: Cotton, Diameter, Perimeter, Maturity ratio.

\footnotetext{
ABSTRACT

Rapid estimations of diameter minus lumen (D-L), and outer perimeter of cotton fibers (P) in microns, as a biological fineness of Egyptian cotton could be calculated with satisfactory levels of accuracy from hair weight (H.W) in $\mathrm{m} / \mathrm{tex}$ and maturity ratio (MR) obtained from Micromat data (new F/MT instrument), using the following equations:

Diameter (microns) $=\sqrt[2]{\frac{\text { Hs } \text { (Standard fineness }}{\text { Circulariy } \times 3.14 \times 1.52}}$

or Final format $($ D) (microns) $=1.205 \sqrt{H s}$

or

Perimeter (microns) $=\sqrt{\frac{4 \times 3.14 \times \mathrm{Hs}}{\text { Circularity } \times 1.52}}$

or Final format $(\mathbf{P})($ microns $)=3.7853 \sqrt{\mathrm{Hs}}$

Results of the current study suggested that more attention should be focused on meaning and measurements of the three values of biological fineness (i.e.) diameter (D), perimeter (P) (microns) and standard fineness (Hs) m/tex. which can be derived from the data obtained from Micromat instrument.
}

\section{INTRODUCTION}

Using fiber cross- sections technique can provide acceptable unbiased values of fiber's perimeter, area of cross- section and degree of wall thickening. Image Analysis is a very efficient and accurate technique to examine a large number of very carefully prepared fiber cross sections. It is a very good tool for research but it is too slow to be of practical use when dealing with large number of samples in commercial purposes, and/or cotton breeding programs, besides the difficulties in preparing fiber cross- sections.

For many purposes, it is necessary to consider only two definitions of fiber fineness:

1) Gravimetric fineness, which is a measure for both fineness and maturity.

2) Biological fineness (Intrinsic fineness), which is expressed by the perimeter of cross- section of the fiber and/or the diameter of the crosssection of the fiber if that section was considered to be circular.

Gravimetric fineness can be related to biological fineness, if the wall thickness or maturity of the fibers is known Ramey (1982). Biological fineness could be measured for the fibers obtained from green bolls just before opening (have a circular cross section) also could be measured using cross- section and image analysis technique. On the other hand the average widths of the fiber, taken at the widest and narrowest points of a convolution near the center of the fiber, could be averaged to obtain an estimate of the fiber ribbon width (diameter). Moreover, the diameter of the swollen fiber treated by $18 \%$ sodium hydroxide can also provide an estimate of fiber diameter. 
Both of cross- section technique and measuring the diameter of unclasped fiber, taken from the green boll before opening are accurate methods for measuring fiber biological fineness parameters but they are suitable for research, being used as reference methods for evaluating the accuracy, reliability and repeatability of the values and estimates obtained from other methods.

During the second stage of cotton fiber growth and development, the successive layers of the secondary wall fill the space inside the primary wall, the proportion filled by the secondary wall layers depends upon the diameter of the fiber and growth conditions as well. The proportion of secondary wall deposition could be measured by the degree of thickening $[\theta]$ Lord (1981).

Degree of thickening $=\theta=\frac{\text { Area of cell wall }}{\text { Area of circle having same perimeter }}$

Lord also stated that degree of thickening = $0.577 \times$ MR. On the other hand, Thibodeaux and Price (1989) used image analyzer programmed automatically to measure cross- section data, the outer perimeter $(\mathrm{P})$ of the fiber as well as its cell wall area (A). Results of (P) and (A) are then used to derive the circularity or degree of thickening $(\theta)$ of the fiber's secondary wall. This is performed according the following Thibodeaux and Price (1989) equation

$$
\theta=\frac{4 \times 3.14 \times A}{P^{2}} \text { where } \mathrm{A}=\text { Cell wall area }
$$

Degree of thickening is defined as the ratio of cell wall area to the area of a circle having the same fiber perimeter. This ratio could be written in the following proposed equation

$$
\text { Degree of thickening }=\theta=\frac{A(\text { cell wall area })}{\left(1 / 2 D^{2}\right) \times 3.14}
$$

According to Thibodeaux

$$
\text { Degree of thickening }=\theta=\frac{4 \times 3.14 \times A}{P^{2}}
$$

Both of the proposed and Thibodeaux equations consist of: a- Area of cell wall $\mathrm{A}=\mathrm{HS} / 1.52$

b- Area of a circle having the same fiber perimeter.

c- The two equations may be converted to the traditional maturity ratio as stated in Lord equation

\section{Degree of thickening $=\theta=0.577 \times M . R$}

Depending on the previous three principals, biological fineness could be calculated from the two equations instead of degree of thickening as follows

1- To calculate the diameter of cotton fiber using the following suggested equation

$$
\begin{aligned}
& 0.577 \times M R=\frac{A}{\left(1 / 2 D^{2}\right) \times 3.14} \\
& \text { or } 1 / 2 D^{2}=\frac{A}{0.577 \times M R \times 3.14} \\
& \text { or } D=\sqrt[2]{\frac{A}{\theta \times 3.14}}
\end{aligned}
$$

2- To calculate the outer perimeter of cotton fibers using Thibodaux equation

$$
\begin{aligned}
& 0.577 x M R=\frac{4 \times 3.14 \times A}{P^{2}} \\
& \text { or } P=\sqrt{\frac{4 \times 3.14 \times A}{0.577 \times M R}}
\end{aligned}
$$

Several methods have been used to measure fineness and maturity of cotton fibers, but the most popular ones are the airflow methods, due to the testing speed and the acceptable and acknowledged accuracy level of their measurements. A double compression fineness and maturity tester (Micromat) which uses air flow principals has been developed to be used routinely to assess cotton fiber's Gravimetric fineness in $\mathrm{m} /$ tex and maturity ratio. Both of them can be estimated independently from the two pressure drop readings. These two readings are subjected to specific equations, which permit the estimation of maturity and fineness parameters. Applying the Micromat data 
to the proposed equations with some modifications in their format and components can enable to obtain estimate values of fiber biological fineness which is very important to cotton breeder and spinner.

Therefore the main objective of this study is

1) To use hair weight (HW) in m/tex Gravimetric fineness, and maturity ratio (MR) obtained from micromat data to estimate biological fineness expressed in diameter and/or perimeter of circular cross section of cotton fiber.

2) To use correlation and regression analysis to compare and evaluate the validity and accuracy of the two values of biological fineness (diameter and outer perimeter).

3) To compare the three values of fiber fineness parameters; diameter, outer perimeter in microns, and standard fineness in $\mathrm{m} /$ tex in relation to fiber bundle strength $(\mathrm{g} / \mathrm{tex})$ and yarn strength.

\section{MATERIALS AND METHODS}

Materials of this study included fifteen Egyptian cottons grown in the miniature trial of Cotton Research Institute during 2000, 2001 and 2002 seasons. The chosen cottons represent the Egyptian cotton categories as follows

1) Five Extra long staple cottons i.e. Giza 45, Giza 87, Giza 70, Giza 88, and Giza $84 \times$ (G.74 × G.68), were grown in seven locations in Delta.

2) Five long staple cottons grown in seven locations in Delta i.e. Giza 85, Giza 86, Giza $89,($ Giza $89 \times$ Giza 87$)$ and Giza $89 \times S^{6}$.

3) Five Long staple cottons grown in six locations in Upper Egypt i.e. Giza 80, Giza 83, Giza 90 and Giza 83.

Aiming to carry out fiber and yarn tests, a sum of 81 , lint cotton samples were taken from the different locations through each of the three growing seasons.

Lint cotton samples were tested using Micromat instrument to determine micronair value, hair weight (m/tex), maturity ratio and maturity percent $(\%)$ as well as using the Stelometer to determine flat bundle strength (g/tex) and elongation $(\%)$, besides producing $60 \mathrm{~s}$ carded yarns from each of the different samples. To be tested for skein strength using Good Brand Tester.

The spinning procedures were carried out in the experimental spinning mill of cotton Res. In- stitute. All fiber and yarn tests were conducted in the labs. of cotton Res. 1nst. according to A.S.T.M. (1998) designations under controlled conditions of $20^{\circ} \mathrm{C} \pm 2$ and $65 \%$ relative humidity.

Standard fineness in $\mathrm{m} / \mathrm{tex}(\mathrm{Hs})$ was calculated from Micromat hair weight (HW) and maturity ratio (MR) as follows

$$
H s \frac{H W(m / t e x)}{M R}
$$

The calculated Hs values were applied to the suggested equation to get estimates for

A- Fiber diameter (D) in microns from the new suggested equation

$$
0.577 M R=\frac{A(m / \text { tex })}{1 / 2 D^{2} \times 3.14}
$$

$$
\text { or } 1 / 2 D^{2}=\frac{A(m / t e x)}{0.577 M R \times 3.14}
$$

or $D($ microns $)=\sqrt[2]{\frac{H s(m / t e x)}{0.577 \times 3.141 .52}}$

$$
\text { or D(microns) }=\sqrt[2]{\frac{H s}{2.7539}}
$$

Final format is

$$
D=1.2051 \sqrt{H s}
$$

B- Fiber outer perimeter $(\mathrm{P})$ microns using this equation

$$
\begin{aligned}
& 0.577 M R=\frac{4 \times 3.14 A}{P^{2}} \\
& \text { or } P \text { (mirons) }=\sqrt[2]{\frac{4 \times 3.14 \times A}{0.577 M R}}
\end{aligned}
$$

Final format is

$$
\boldsymbol{P}=3.7853 \sqrt{\mathrm{Hs}}
$$

$\left[1.52 \mathrm{gm} / \mathrm{cm}^{3}=\right.$ Density of fiber minus lumen (cellulose density). (4)] 


\section{Statistical analysis}

Simple correlation and stepwise regression analysis were employed to study the relationships between the three fineness estimates and their power to predict fiber bundle strength and yarn strength.

\section{RESULT AND DISCUSSION}

\section{A- Correlation coefficients}

The diameter, minus lumen obtained from the suggested equation and the outer perimeter of cotton fibers obtained from Thibodeaux equation showed very high and strong relationship. A perfect linear positive correlations close to 1.00 , was observed. The obtained correlation coefficients were $0.99997,1.000$ and 1.0000 through the seasons 2000, 2001 and 2002 respectively, Table (1).

The perfect positive correlation coefficients through the three seasons mean that the proposed equation has the same accuracy of Thibodeaux equation, while the repeatability of complete correlation at 1.0 through the three seasons ensures the relationship between the two variables which are expected due to the mathematical relationship between the diameter and perimeter of the circle which equal 3.14 .

Table 1. Correlations between values of perimeter and diameter and standard fineness $(\mathrm{Hs})$ through seasons 2000, 2001 and 2002.

\begin{tabular}{|lccc|}
\hline \multicolumn{1}{|c|}{$\begin{array}{l}\text { Fineness } \\
\text { parameters }\end{array}$} & 2000 & 2001 & 2002 \\
\hline $\begin{array}{l}\text { Diameter } \times \\
\text { perimeter }\end{array}$ & $0.99997^{* *}$ & $1.0000^{* *}$ & $1.0000^{* *}$ \\
$\begin{array}{l}\text { Diameter } \times \\
\text { stand fine- } \\
\text { ness }\end{array}$ & $0.91928^{* *}$ & $0.9841^{* *}$ & $0.8951^{* *}$ \\
$\begin{array}{l}\text { Perimeter } \times \\
\text { stand fine- } \\
\text { ness }\end{array}$ & $0.9197 * *$ & $0.9840^{* *}$ & $0.8951^{* *}$ \\
\hline
\end{tabular}

These results are generally due to the correctness and/or to the equalization of the equation that calculates diameter minus lumen (microns) as compared with Thib. Equation to calculate outer perimeter (microns).

\section{B- Regression coefficients}

\section{1- Ratio of perimeter to expected diameter}

Figure (1) reveals, the regression lines and equations for outer perimeter to the expected diameter minus lumen of cotton fiber through the seasons 2000, 2001 and 2002.

The regression coefficients were very close, being 3.118, 3.0707 and 3.1383. Through the seasons 2000, 2001 and 2002, respectively with an average of 3.1093 Table (2). The above regression coefficients for outer perimeter (microns) to the expected fiber diameter minus lumen (microns) are in accordance and agreement with the mathematical relationship perimeter and diameter being $22 / 7$ or 3.1416 .

\section{Perimeter $=$ Diameter $\times 3.1416$}

\section{2- Ratio of diameter to expected perimeter}

Figure (2) reveals the regression lines and coefficients for fiber diameter minus lumen (microns) upon outer perimeter (microns) of cotton fibers through the seasons 2000, 2001 and 2002. The regression coefficients were $0.3202,0.3210$ and 0.3178 through the seasons 2000, 2001 and 2002 respectively, with an average of 0.3199 Table (2).

Table 2. Mean of perimeter, diameter in microns and its regression coefficients for P/D-L and D-L/P.

\begin{tabular}{|ccccc|}
\hline Seasons & $\begin{array}{c}\text { Perimeter } \\
\text { mean }\end{array}$ & $\begin{array}{c}\text { Diameter } \\
\text { mean }\end{array}$ & Regression & Regression \\
\hline 2000 & 50.45 & 16.06 & 3.118 & 0.3202 \\
2001 & 47.39 & 15.09 & 3.0707 & 0.3210 \\
2002 & 46.70 & 14.87 & 3.1384 & 0.3178 \\
\hline Mean & 48.18 & 15.34 & 3.1093 & 0.3199 \\
\hline
\end{tabular}

The previous regression coefficients for fiber diameter minus lumen (microns) to the expected outer perimeter (microns) are in accordance and agreement with the mathematical relationship diameter and perimeter being $7 / 22$ or 0.31818 . 


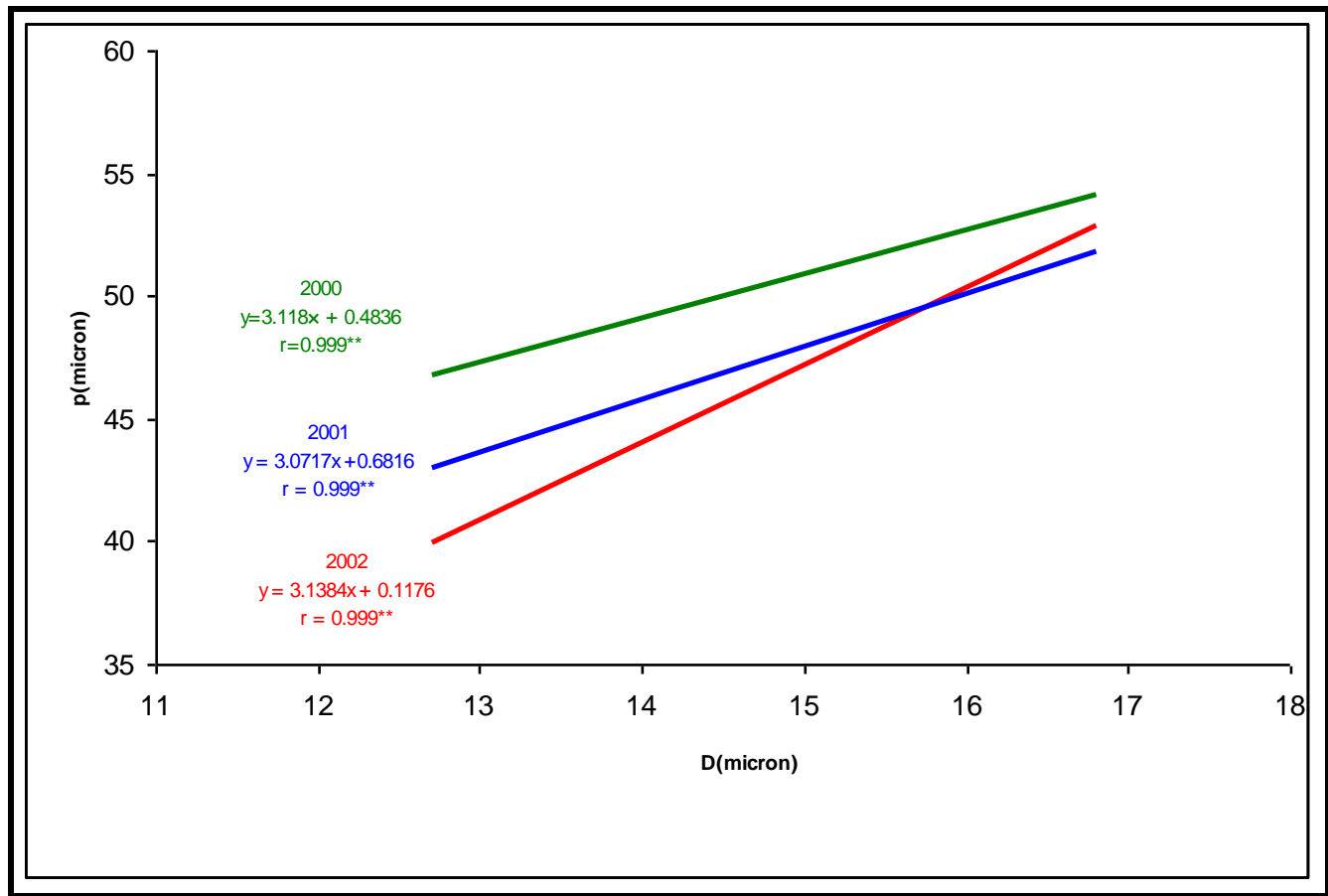

Fig 1. The relationship between Perimeter (P) and Diamter (D) measurments of the Egyptian cotton

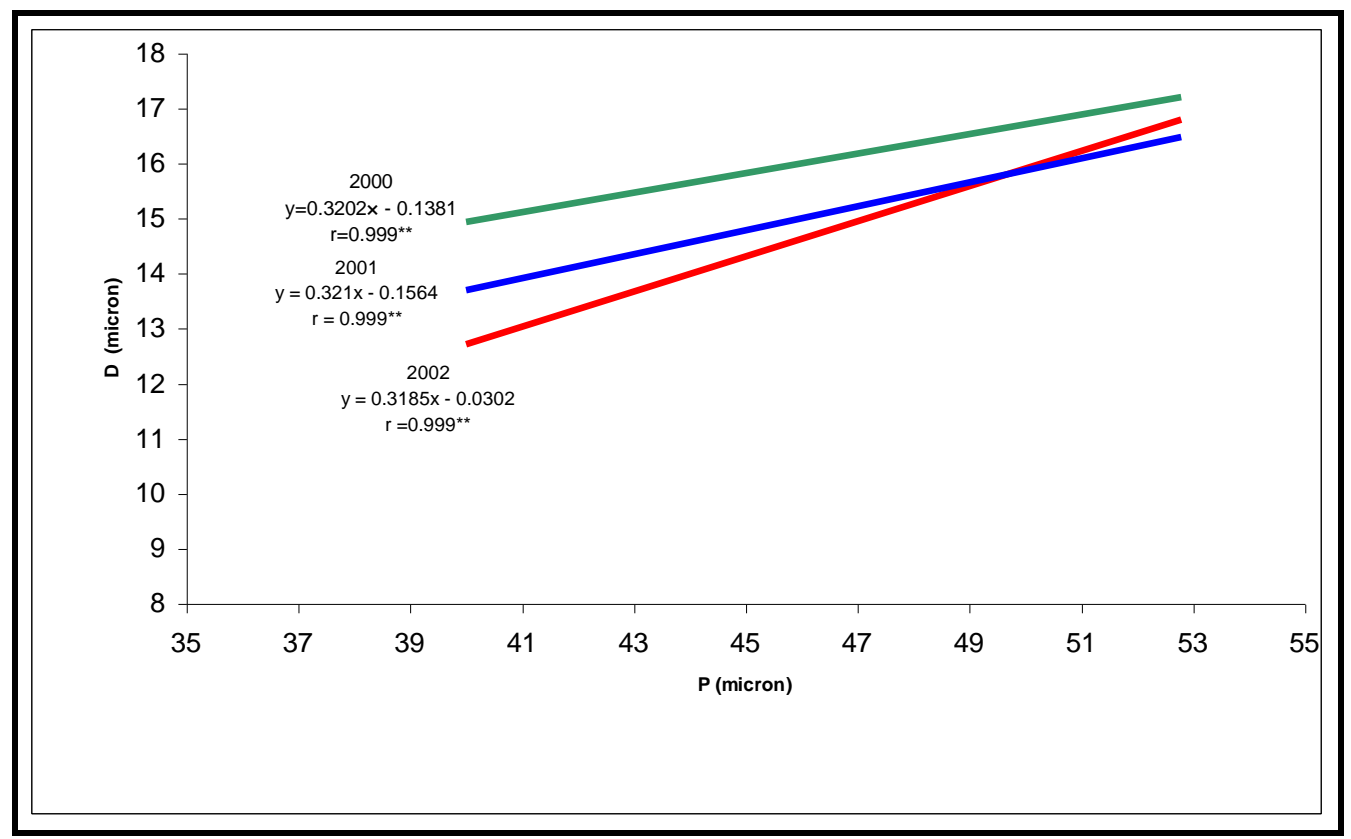

Fig. 2. The relationship between Diamter (D) and Perimeter (P) measurments of the Egyptian cotton 


\section{Diameter $=$ perimeter $\times 0.31816$}

\section{3- Correlation coefficients among the three bio- logical fineness measurements:}

Table (1) reveals the correlations among the three biological fineness parameters of cotton fiber.

Positive and highly significant correlations were found between:

1) Fiber diameter minus lumen (microns) and standard fineness $(\mathrm{m} / \mathrm{tex})$, the correlations were 0.9197 and .9840 and 0.8951 through the seasons 2000, 2001 and 2002, respectively.

2) Fiber outer perimeter (microns) and standard fineness $(\mathrm{m} / \mathrm{tex})$, the correlations were $0.9196,0.9840$ and 0.8951 through the seasons 2000, 2001 and 2002, respectively.

These results are generally satisfying, since positive correlations among the three calculated biological fineness parameters mean that fiber diameter minus lumen will tend to increase as the fiber outer perimeter increases, and as the fiber standard fineness increases and vise versa.

This would be of great benefits to the efforts that aim to improving cotton fiber quality during cotton breeding programs, since it would provide acceptable and reliable estimates of fiber diameter, perimeter and standard fineness, which are known to be heritable Hequet (1988).

\section{Predictive power of the three fiber fineness measurements}

The current study extended to study the predictive power of the calculated fiber diameter (minus lumen) in microns, obtained from the suggested equation, cotton fiber outer perimeter in microns calculated from Thib. equation and standard fineness $(\mathrm{m} / \mathrm{tex})$ in predicting fiber bundle strength (g/tex), and yarn strength Table (3) as one of the main objectives of textile manufacturing.

Data in Table (3) show highly significant negative correlations between fiber fineness and each of fiber bundle strength (g/tex) and yarn strength. The recorded correlation coefficients of fiber perimeter and diameter were nearly the same for both of fiber and yarn strength being -0.8822 , -0.7458 and -0.846 for the three seasons respectively, however standard fineness showed slightly different correlation coefficients being -0.9051 , -0.6919 and -0.7912 for the three seasons respectively. On the other hand the negative relationship of fiber fineness with fiber and yarn strength indicates that fiber and yarn strength tend to be increased as fiber diameter, perimeter and standard fineness (Hs) decreased. This behavior could be attributed to the factors affecting both bundle and yarn strength, individual fiber strength, individual fiber elongation, the number of fiber in the bundle or in the cross- section, besides the factors concerning yarn structure as yarn count, twist function and cohesion forces...etc.

Table 3. Correlation between perimeter, diameter and standard fineness on the level of bundle strength and yarn strength.

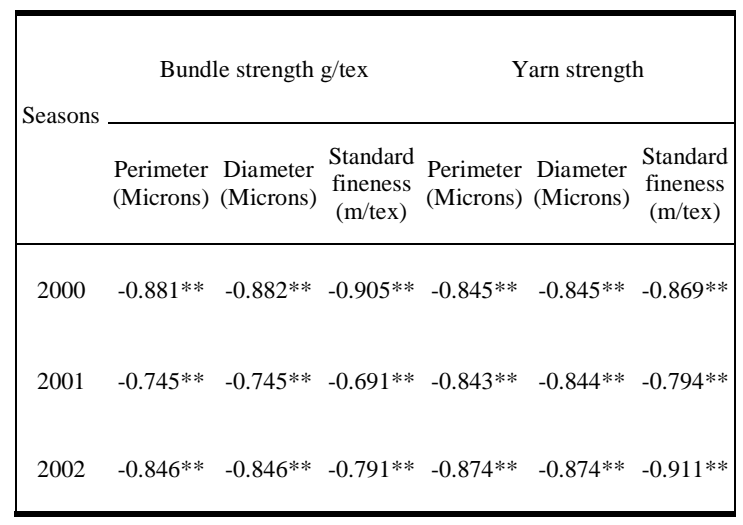

Results of the current study suggested that more attention should be focused on measuring and measurements of the three values of biological fineness, diameter (D) and perimeter $(\mathrm{P})$ of circle (microns) and standard fineness (Hs) $\mathrm{m} / \mathrm{tex}$, which can be derived from the data obtained from Micromat. 


\section{REFERENCES}

A.S.T.M. (1998). American Society for Testing and Materials Standards on Textile Materials. D: 1445-75. Philadelphia 3, Pa, U.S.A.

Hequet E. (1988). Influence of soil type and planting date on yarn quality in Chad. Proceeding International Cotton Conference, Bremen p. 9. Lord E. (1981). The Origin and Assessment of Cotton Fiber Maturity. pp: 1-27. Technical
Research Division, International Institute for Cotton, Manchester, England.

Ramey H.H.Jr. (1982). The Meaning and Assessment of Cotton Fiber Fineness. pp: 1-19. Technical Research Division, International Institute for Cotton, Manchester, England.

Thibodeaux, D.P. and J.B. Price (1989). Reference method for determination of the maturity of cotton fiber. Melliand Textiber. 70: 243246. 